\title{
Ultrasonographic assessment of transitional cell carcinoma of the urinary bladder in dogs: a perspective of tumor size change
}

\author{
Seungji Kim, Yongbaek Kim, Wanhee Kim, Mincheol Choi, Junghee Yoon* \\ College of Veterinary Medicine and the Research Institute for Veterinary Science, Seoul National University, Seoul 08826, Korea
}

(Received: March 24, 2017; Revised: June 1, 2017; Accepted: July 4, 2017)

\begin{abstract}
Study purposes were to evaluate ultrasonographic characteristics of transitional cell carcinoma (TCC) and quantitate bladder tumor size in dogs. Heterogeneous mass, wall involvement, and broad-based attachment were significantly associated with TCC, but not prominently the trigone region. Mass size evaluation revealed a significant correlation between progressive disease (PD) in TCC patients with piroxicam therapy. Largest diameter of target lesion/ body weight $(\mathrm{cm} / \mathrm{kg})$ ratio showed a high mean value in PD. A value $>0.3$ was associated with $\mathrm{PD}$ with $83 \%$ sensitivity and $66 \%$ specificity. The results suggest that ultrasonography can provide evidence for diagnosing and predicting a prognosis for TCC.
\end{abstract}

Keywords: dogs, mass size, transitional cell carcinoma, urinary bladder, ultrasonography

Urinary bladder cancer is approximately $2 \%$ of all reported malignancies in dogs. The estimates accounts for more than 70 million dogs in the United States. Among these cancers, invasive transitional cell carcinoma (TCC) is the most common type of canine urinary bladder cancer [6, 14]. Canine TCC is complicated in some reasons, and is most often located in the trigone or urethral involvement. Concurrent with thickened bladder wall causes extramural compression of ureterovesical junction leading to dysuria and partial or complete obstruction of the urinary tract [8]. This type of cancer metastasizes widely; $78 \%$ of dogs with these cancers have T2 tumors (invading bladder wall) and $20 \%$ have T3 tumors (invading neighboring organs) at the time of diagnosis according to World Health Organization (WHO) criteria for staging canine bladder tumors [6].

Neoplastic cells may be present in the urine of $30 \%$ of dogs with TCC. However, neoplastic cells often are indistinguishable from reactive epithelial cells associated with inflammation [13]. Therefore, the definitive diagnosis of TCC requires histopathologic confirmation of tissues obtained by cystotomy, cystoscopy, or catheter biopsy [7].

Urinary tract imaging such as ultrasonography is a preferred method seeing as it can be accurately performed in awake dogs in a timely and cost-effective fashion [2]. In ultrasonography, TCC shows typically irregular bladder wall mass with a broad-based attachment projecting into the urinary bladder lumen. The echogenicity is often mixed and has an overall appearance that can be echogenic, and partly min- eralized [9]. Furthermore, ultrasonography is used to assess the location of tumors, to provide prognostic information, and to map and measure TCC masses in order to subsequently monitor response to medical therapy [4, 5, 11]. Yet, these studies have limitations such as focusing only on large breed dogs and lack of clinical utility of tumor size approach to detect response to treatment during serial recheck.

The purposes of this study were to re-evaluate ultrasonographic characteristics including tumor size change in small to middle breed dogs (less than $12 \mathrm{~kg}$ ) and quantitate the bladder tumor size to seek any diagnostic relevance or help predicting prognosis of TCC.

Twenty-one dogs confirmed with bladder mass lesions by histopathology and/or cytology were included in this study with reviewing medical records at the Seoul National University Veterinary Medical Teaching Hospital from 2012 through 2016. They are divided into TCC group and Non-TCC group. TCC group was those diagnosed with transitional cell carcinoma $(\mathrm{n}=11)$. And Non-TCC group was consisted of polypoid cystitis $(n=5)$ and bacterial cystitis $(n=5)$ patients. Detailed ultrasonographic characteristics were recorded for all cases including echogenicity, wall invasion, margination, morphology, location and blood signal on color Doppler image.

To evaluate the size of the masses, urinary bladder was adequately distended with normal saline injection through urinary catheter. Tumor size was determined with a largest diameter of the target lesion before and after piroxicam (Crown Pharm, Korea) therapy. To evaluate the clinical sig-

*Corresponding author

Tel: +82-2-880-1265, Fax: +82-2-876-8662

E-mail: heeyoon@snu.ac.kr 
nificance of measurement differences, Response Evaluation Criteria in Solid Tumours (RECIST) criteria was used as a guideline for assessing response. According to the RECIST criteria, complete response is disappearance of the target lesion, partial response is at least at $30 \%$ decrease in the largest diameter of the target lesion, progressive disease is at least a $20 \%$ increase in the largest diameter of the target lesion, and stable disease is neither sufficient shrinkage nor increase in the largest diameter to quantify for a partial response or progressive disease [12].

The largest diameter of tumor was indexed to body weight before therapy (modified mass size), and was calculated using the formula: largest diameter of target lesion/body weight to quantitate the bladder tumor size regardless to the size of the patients. And the authors sought correlation between modified mass size $(\mathrm{cm} / \mathrm{kg})$ and response to the therapy which are previously recorded (e.g., partial response, progressive disease and stable disease).

All statistical tests were performed using SPSS (IBM SPSS Statistics for Windows, ver. 23.0; IBM, USA). Data were expressed as mean $\pm \mathrm{SD}$, and value of $p<0.05$ were considered significant. Statistical significance for difference was calculated with Mann-Whitney test.

Dogs weighed between 2.1 and $11.7 \mathrm{~kg}$ (mean weight, 5.0 $\mathrm{kg}$ ) and ranged in age between 8 to 16 years (mean age, 11.5 years). There were 7 spayed females, 4 neutered males in TCC group, otherwise, 6 of neutered males and 4 of spayed females were counted in Non-TCC group. There was no significance difference in age, body weight, sex, and breed predisposition between TCC and Non-TCC groups. Breeds represented in TCC groups in descending order were three Poodle, 2 each of Maltese and Shih-Tzu, one each of Schnauzer, Miniature Pinscher, Scottish terrier and Pomeranian. Chief complaints, which likely resulted from these diseases were hematuria, dysuria, and pollakiuria. The mean follow-up period was 61.6 days (range, 12-201 days). Results of this study reported here consisted with Scottish terrier and female sex are predisposing factor. A Scottish terrier was counted in TCC group although it is not a popular breed in this country and these results targeted at a limited population.

Canine TCC is highly invasive and mostly at the stages of $\mathrm{T} 2$ at the time of diagnosis, therefore it is essential to evaluate tissue structures adjacent to the bladder mass in TCC suspected patients. On ultrasonography, there was three-layered structures, two hyperechoic parallel thin layers and a hypoechoic layer between the two hyperechoic layers which represents muscular layer. Ultrasonography could reliably predict bladder wall involvement and was significantly associated with histological results (sensitivity $93 \%$ and specificity 92\%) [4]. According to WHO staging system of TCC, 6 dogs of TCC groups were T2N0M0, 2 dogs were T1N0M0 and one dog was T2N1M0. The remaining one dog was excluded from the evaluation of bladder wall invasion due to poor imaging, but regional or distance metastasis was not detected. No statistical correlation between severity of staging and
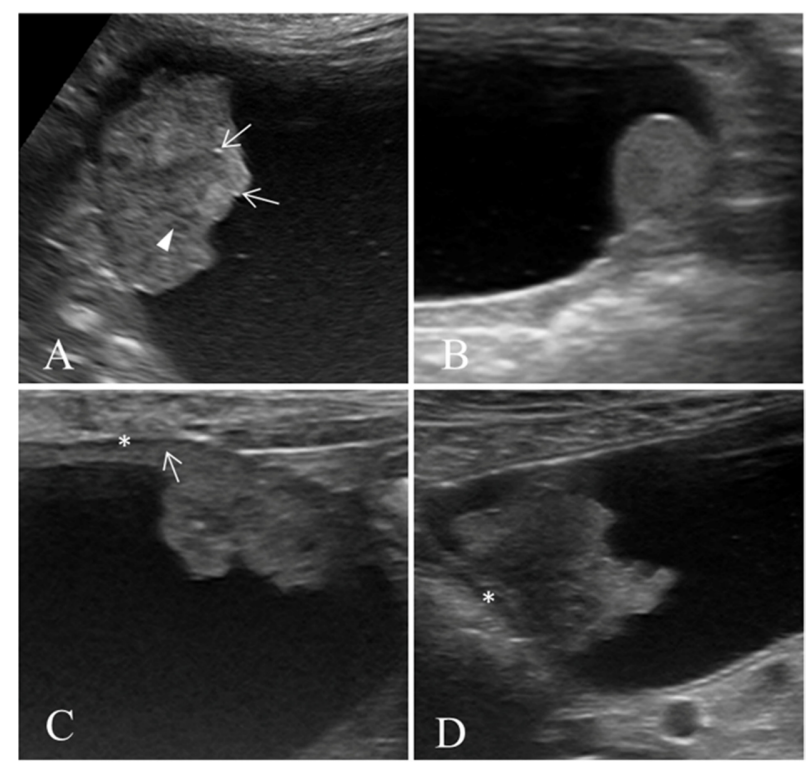

Fig. 1. Ultrasonographic images of bladder mass with heterogeneous (A) or homogeneous (B) echo patterns. Heterogeneous mass contains some hypoechoic (arrowhead) and hyperechoic (arrows) areas. (C) Muscular involvement case of transitional cell carcinoma. The mass is extended into the hypoechoic muscular layer (asterisk) and discontinuation of wall layer (arrow), which indicates involvement of wall invasion. (D) Non-involvement of wall invasion. Mass is apart from the hypoechoic layer (asterisk).

increase in mass size change was detected in this study. Although one patient revealed regional lymph node metastasis on histopathology, there was no evidence of metastasis at the ultrasonographic examination during the several recheck periods. As a result, all patients had suspected TCC on urine sediment examination and no evidence of metastasis at the time of diagnosis. Therefore, piroxicam with the least effects and effective against transitional cell carcinoma was prescribed.

Wall involvement along with heterogeneous mass and trigone location in TCC patients was significantly associated with shorter survival times [4]. In this study, heterogeneous echo pattern and bladder wall involvement of mass (Fig. 1) were significant in TCC group which are sentinel findings for distinguishing TCC from other benign non-TCC masses. It is of use not only providing prognostic indicators but also potential parameters in diagnosing TCC.

Regarding distributions of mass in TCC group in this study, the frequency was the same in trigone region and dorsal wall, although it is widely known to be affected in trigone region in TCC. In a previous report, dorsal wall location of the TCC was the second most common affected site followed by trigone region [9]. Moreover, considering clinical significance of trigone affected TCC which eventually leads to urinary tract obstruction with complication, other distributions of mass could be underestimated as it could be subclinical or incidental findings. Therefore, trigone region should be considered as a negative prognostic factor in TCC patients, not the per- 
Table 1. Ultrasonographic findings of TCC and Non-TCC patients

\begin{tabular}{|c|c|c|}
\hline Ultrasonography & TCC & Non-TCC \\
\hline \multicolumn{3}{|l|}{ Echo pattern } \\
\hline Heterogeneous & $8^{*}$ & 3 \\
\hline Homogenous & 2 & 6 \\
\hline \multicolumn{3}{|l|}{ Bladder wall invasion } \\
\hline Invasion & $8^{*}$ & 3 \\
\hline Noninvasion & 2 & 7 \\
\hline \multicolumn{3}{|l|}{ Distribution } \\
\hline Trigone & 4 & 2 \\
\hline Non trigone & 9 & 9 \\
\hline \multicolumn{3}{|l|}{ Margination } \\
\hline Irregular & 10 & 9 \\
\hline Smooth & 1 & 1 \\
\hline \multicolumn{3}{|l|}{ Morphology } \\
\hline Broad based & $7 *$ & 2 \\
\hline Pedunculated & 1 & 5 \\
\hline \multicolumn{3}{|c|}{ Blood signal on Doppler } \\
\hline Moderate & 3 & 2 \\
\hline Mild or absent & 6 & 6 \\
\hline
\end{tabular}

Statistical differences were calculated with Mann-Whitney test. ${ }^{*} p<0.05$.

Table 2. Difference in response to piroxicam therapy and mean, SD of largest diameter of target lesion/body weight

\begin{tabular}{lcc}
\hline \hline & Progressive disease & $\begin{array}{c}\text { Stable disease and } \\
\text { partial response }\end{array}$ \\
\hline TCC $(\mathrm{n}=9)$ & $6^{*}$ & 3 \\
Non-TCC $(\mathrm{n}=8)$ & $0.59 \pm 0.43$ & $0.29 \pm 0.08$ \\
& 0 & 8 \\
\hline
\end{tabular}

Statistical differences were calculated with Mann-Whitney test. $* p<0.05$.

spective of more affected site (Table 1).

There are some limitations in diagnosing TCC because of poor diagnostic value of urine sediment (up to 30\%) and invasiveness of histopathologic definitive diagnosis [7]. In this study, TCC group showed progressive disase significantly although patients were going on piroxicam therapy (Table 2). And the majority (6/8) of patients is a stable disease in non-TCC group. Therefore, further short term followup is required in TCC suspected patients (Fig. 2).

Several studies are conducted about size of the bladder tumor $[4,5,10]$, but they are mostly focused on large breed dogs and no quantitative evaluation considering body size has been studied. On initial ultrasonographic examination, modified tumor size value $(\mathrm{cm} / \mathrm{kg})$ had a tendency to be much higher on progressive disease patients. The value over $0.3(\mathrm{~cm} / \mathrm{kg})$ was associated with progressive disease in TCC with a sensitivity of $83 \%$ and specificity of $66 \%$.

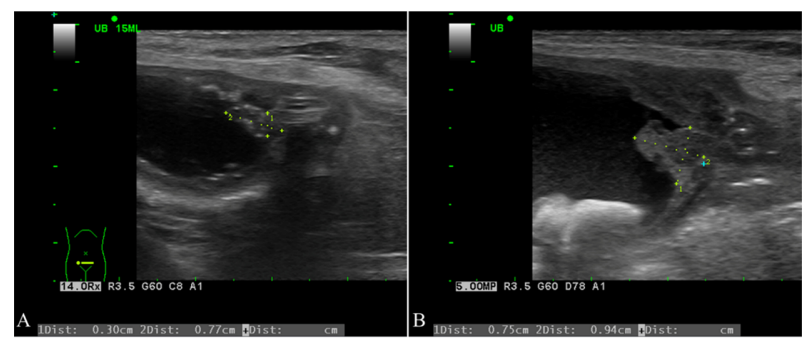

Fig. 2. Ultrasonographic images on sequential visits obtained from a 12 years old patient with chief complaint of hematuria. Later, this patient had a surgery of partial cystotomy and diagnosed with TCC from histopathologic result. Bladder was moderately distended for well-visualization of masses with $15 \mathrm{~mL}$ and $20 \mathrm{~mL}$ of saline, respectively. (A) On initial visit, measurement of the mass was $0.77 \times 0.30 \mathrm{~cm}$, and modified size value of maximal diameter was $0.35(\mathrm{~cm} / \mathrm{kg})$. (B) 142 days after piroxicam therapy. The maximal diameter exceeded $20 \%$ of initial maximal diameter of target lesion $(0.94 \times 0.75 \mathrm{~cm})$ which manifests that this lesion shows characteristics of progressive disease. Note modified size value exceeds $0.3(\mathrm{~cm} / \mathrm{kg})$.

There are multiple medical therapies suggested in previous studies in TCC, which usually consists of chemotherapy, cyclooxygenase inhibitor and combination of these [1, 3-6]. Piroxicam therapy is generally well tolerated in TCC patients even if tumor remission did not occur, owners subjectively noted improved quality of life $[1,2]$. However, different or additional therapies should be required if cancer is progressive or unacceptable complication occurs.

The limitation of study is from the retrospective nature. This study provides variable parameters such as all different recheck periods, different operators on ultrasonographic examination, and no quantitative bladder distension considering one's size. The study samples are relatively small number of populations, further study among large population should be done. Additional evaluation is needed for response to the medication therapy focusing on clinical relevance such as clinical signs and quality of life, since ultrasonography alone is an inaccurate way to evaluate disease progression.

In conclusion, ultrasonographic tumor size evaluation could be helpful in both diagnosing and predicting prognosis in canine TCC. Heterogeneous echogenicity, bladder wall involvement, broad based attachment on ultrasonography and progressive condition of mass size change in follow up examination during piroxicam therapy represent strong association with TCC. Initial mass size over $0.3(\mathrm{~cm} / \mathrm{kg})$ is also prone to be progressive in size despite of the therapy.

\section{References}

1. Boria PA, Glickman NW, Schmidt BR, Widmer WR, Mutsaers AJ, Adams LG, Snyder PW, DiBernardi L, de Gortari AE, Bonney PL, Knapp DW. Carboplatin and piroxicam therapy in 31 dogs with transitional cell carcinoma of the urinary bladder. Vet Comp Oncol 2005, 3, 73-80.

2. Fulkerson CM, Knapp DW. Management of transitional cell 
carcinoma of the urinary bladder in dogs: a review. Vet $\mathrm{J}$ 2015, 205, 217-225.

3. Greene SN, Lucroy MD, Greenberg CB, Bonney PL, Knapp DW. Evaluation of cisplatin administered with piroxicam in dogs with transitional cell carcinoma of the urinary bladder. J Am Vet Med Assoc 2007, 231, 1056-1060.

4. Hanazono K, Fukumoto S, Endo Y, Ueno H, Kadosawa T, Uchide T. Ultrasonographic findings related to prognosis in canine transitional cell carcinoma. Vet Radiol Ultrasound 2014, 55, 79-84.

5. Hume C, Seiler G, Porat-Mosenco Y, Caceres A, Shofer F, Sorenmo K. Cystosonographic measurements of canine bladder tumours. Vet Comp Oncol 2010, 8, 122-126.

6. Knapp DW, Glickman NW, DeNicola DB, Bonney PL, Lin TL, Glickman LT. Naturally-occurring canine transitional cell carcinoma of the urinary bladder A relevant model of human invasive bladder cancer. Urol Oncol 2000, 5, 47-59.

7. Knapp DW, Glickman NW, Widmer WR, DeNicola DB, Adams LG, Kuczek T, Bonney PL, DeGortari AE, Han C, Glickman LT. Cisplatin versus cisplatin combined with piroxicam in a canine model of human invasive urinary bladder cancer. Cancer Chemother Pharmacol 2000, 46, 221226.

8. Knapp DW, McMillan SK. Tumors of the urinary system. In: Withrow SJ, Vail DM, Page RL (eds.). Withrow and MacEwen's Small Animal Clinical Oncology. 5th ed. pp. 572-582, Elsevier Saunders, Saint Louis, 2013.

9. Knapp DW, Richardson RC, Chan TCK, Bottoms GD, Widmer WR, DeNicola DB, Teclaw R, Bonney P, Kuczek
T. Piroxicam therapy in 34 dogs with transitional cell carcinoma of the urinary bladder. J Vet Intern Med 1994, 8, 273-278.

10. Léveillé R, Biller DS, Partington BP, Miyabayashi T. Sonographic investigation of transitional cell carcinoma or the urinary bladder in small animals. Vet Radiol Ultrasound 1992, 33, 103-107.

11. Mohammed SI, Bennett PF, Craig BA, Glickman NW, Mutsaers AJ, Snyder PW, Widmer WR, DeGortari AE, Bonney PL, Knapp DW. Effects of the cyclooxygenase inhibitor, piroxicam, on tumor response, apoptosis, and angiogenesis in a canine model of human invasive urinary bladder cancer. Cancer Res 2002, 62, 356-358.

12. Naughton JF, Widmer WR, Constable PD, Knapp DW. Accuracy of three-dimensional and two-dimensional ultrasonography for measurement of tumor volume in dogs with transitional cell carcinoma of the urinary bladder. Am J Vet Res 2012, 73, 1919-1924.

13. Therasse P, Arbuck SG, Eisenhauer EA, Wanders J, Kaplan RS, Rubinstein L, Verweij J, Van Glabbeke M, van Oosterom AT, Christian MC, Gwyther SG. New guidelines to evaluate the response to treatment in solid tumors. J Natl Cancer Inst 2000, 92, 205-216.

14. Valli VE, Norris A, Jacobs RM, Laing E, Withrow S, Macy D, Tomlinson J, McCaw D, Ogilvie GK, Pidgeon G, Henderson RA. Pathology of canine bladder and urethral cancer and correlation with tumour progression and survival. J Comp Pathol 1995, 113, 113-130. 